\title{
ITERATIVE COMPUTATION FOR SOLVING CONVEX OPTIMIZATION PROBLEMS OVER THE SET OF COMMON FIXED POINTS OF QUASI-NONEXPANSIVE AND DEMICONTRACTIVE MAPPINGS
}

\author{
Thierno M. M. Sow \\ Université Amadou Mahtar Mbow, Dakar, Senegal
}

\begin{abstract}
In this paper, a new iterative method for solving convex minimization problems over the set of common fixed points of quasi-nonexpansive and demicontractive mappings is constructed. Convergence theorems are also proved in Hilbert spaces without any compactness assumption. As an application, we shall utilize our results to solve quadratic optimization problems involving bounded linear operator. Our theorems are significant improvements on several important recent results.

Keywords:Fixed point algorithm, Convex minimization problem, Quasi-nonexpansive mapping, Demicontractive mappings.
\end{abstract}

\section{Introduction}

Let $H$ be a real Hilbert space, $K$ be a nonempty subset of $H$. A map $T: K \rightarrow K$ is said to be Lipschitz if there exists an $L \geq 0$ such that

$$
\|T x-T y\| \leqslant L\|x-y\|, \forall x, y \in K,
$$

if $L<1, T$ is called contraction and if $L=1, T$ is called nonexpansive.

We denote by $\operatorname{Fix}(T)$ the set of fixed points of the mapping $T$, that is $\operatorname{Fix}(T):=$ $\{x \in D(T): x=T x\}$. We assume that Fix $(T)$ is nonempty. If $T$ is nonexpansive mapping, it is well known $\operatorname{Fix}(T)$ is closed and convex. A map $T$ is called quasinonexpansive if $\|T x-p\| \leq\|x-p\|$ holds for all $\mathrm{x}$ in $\mathrm{K}$ and $p \in \operatorname{Fix}(T)$. The

Received August 15, 2019. accepted April 04, 2021.

Communicated by Dragana Cvetković-Ilić

Corresponding Author: Thierno M.M. Sow,Université Amadou Mahtar Mbow, Dakar, Senegal |

E-mail: thierno.sow@uam.edu.sn

2010 Mathematics Subject Classification. 47H09, 47H10, 65C25.

(C) 2021 by University of Niš, Serbia | Creative Commons License: CC BY-NC-ND 
mapping $T: K \rightarrow K$ is said to be firmly nonexpansive, if

$$
\|T x-T y\|^{2} \leq\|x-y\|^{2}-\|(x-y)-(T x-T y)\|^{2}, \forall x, y \in K .
$$

A mapping $T: K \rightarrow H$ is called k-strictly pseudo-contractive if there exists $k \in[0,1)$ such that

$$
\|T x-T y\|^{2} \leq\|x-y\|^{2}+k\|x-y-(T x-T y)\|^{2}, \forall x, y \in K .
$$

A map $T$ is called $k$-demi-contractive if $\operatorname{Fix}(T) \neq \varnothing$ and for $k \in[0,1)$, we have

$$
\|T x-p\|^{2} \leq\|x-p\|^{2}+k\|x-T x\|^{2}, \forall x \in K, \quad p \in F i x(T) .
$$

We note that the following inclusions hold for the classes of the mappings:

firmly nonexpansive $\subset$ nonexpansive $\subset$ quasi-nonexpansive $\subset k$-strictly pseudocontractive $\subset k$-demi-contractive.

The function $T$ in the following example is $k$-demi-contractive mapping but is not a $k$-strictly pseudo-contractive mapping.

Example 1.1. Let $H=\mathbb{R}$ and $K=[-1,1]$. Define $T: K \rightarrow K$ by

$$
T x=\left\{\begin{array}{l}
\frac{2}{3} x \sin \left(\frac{1}{x}\right), \quad x \neq 0 \\
0 \quad x=0 .
\end{array}\right.
$$

Clearly $\operatorname{Fix}(T)=\{0\}$. For $x \in K$, we have

$$
\begin{aligned}
|T x-0|^{2} & =\left|\frac{2}{3} x \sin \left(\frac{1}{x}\right)\right|^{2} \\
& \leq\left|\frac{2}{3} x\right|^{2} \\
& \leq|x|^{2} \\
& \leq|x-0|^{2}+k|x-T x|^{2} \quad \forall k \in[0,1) .
\end{aligned}
$$

Thus $T$ is $k$ demi-contractive for $k \in[0,1)$. To see that $T$ is not $k$ strictly pseudocontractive, choose $x=\frac{2}{\pi}$ and $y=\frac{2}{3 \pi}$, then

$$
|T x-T y|^{2}>|x-y|^{2}+k|x-y-(T x-T y)|^{2} .
$$

Hence, $T$ is not $\mathrm{k}$ strictly pseudo-contractive mapping for $k \in[0,1)$.

The function $T$ in the following example is $k$-demi-contractive mapping but is not not quasi-nonexpansive.

Example 1.2. Let $f$ be a real function defined by $f(x)=-x^{2}-x$; it can be seen that $f:[-2,1] \rightarrow[-2,1]$. This function is demicontractive on $[-2,1]$ and continuous. It is not quasi-nonexpansive and is not pseudocontractive on $[-2,1]$ (check for instance the condition of pseudocontractivity for $x=-1.5$ and $y=-0.6$ ). 
For several years, the study of fixed point theory for nonlinear mappings has attracted, and continues to attract the interest of several well known mathematicians (see, $[9,10,13,4])$.

Interest in the study of fixed point theory for nonlinear mappings stems, perhaps, mainly from its usefulness in real-world applications such as Game Theory and Market Economy and in other areas of mathematics, such as in Non-Smooth Differential Equations and Differential Inclusions, Optimization theory.

Let $K$ be a nonempty, closed convex subset of $H$. The nearest point projection from $H$ to $K$, denoted by $P_{K}$ assigns to each $x \in H$ the unique $P_{K} x$ with the property

$$
\left\|x-P_{K} x\right\| \leq\|y-x\|
$$

for all $y \in K$. It is well known that $P_{K}$ satisfies

$$
\left\langle x-y, P_{K} x-P_{K} y\right\rangle \geq\left\|P_{K} x-P_{K} y\right\|^{2}
$$

for all $y \in H$ and

$$
\left\langle P_{K} z-y, z-P_{K} z\right\rangle \geq 0
$$

for all $z \in K$ and $y \in H$.

An operator $A: K \rightarrow H$ is called monotone if

$$
\langle A x-A y, x-y\rangle_{H} \geq 0, \quad \forall x, y \in K,
$$

$A$ is called $k$-strongly monotone if there exists $k \in(0,1)$ such that for each $x, y \in H$ such that

$$
\langle A x-A y, x-y\rangle_{H} \geq k\|x-y\|^{2} .
$$

An operator $A: H \rightarrow H$ is said to be strongly positive bounded if there exists a constant $c>0$ such that

$$
\langle A x, x\rangle_{H} \geq c\|x\|^{2}, \quad \forall x \in H .
$$

Remark 1.1. From the definion of $A$, we note that strongly positive bounded linear operator $A$ is a $\|A\|$-Lipchitzian and $c$-strongly monotone operator.

Definition 1.1. Let $H$ be a real Hilbert space. A function $g: H \rightarrow \mathbb{R}$ is said to be $\alpha$-strongly convex if there exists $\alpha>0$ such that for every $x, y \in H$ with $x \neq y$ and $\beta \in(0,1)$, the following inequality holds:

$$
g(\beta x+(1-\beta) y) \leq \beta g(x)+(1-\beta) g(y)-\alpha\|x-y\|^{2} .
$$


Lemma 1.1. Let $H$ be a real Hilbert space and $g: H \rightarrow \mathbb{R}$ a real-valued differentiable convex function. Assume that $g$ is strongly convex. Then the differential map $\nabla g: H \rightarrow H$ is strongly monotone, i.e., there exists a positive constant $k$ such that

$$
\langle\nabla g(x)-\nabla g(y), x-y\rangle \geq k\|x-y\|^{2} \forall x, y \in H .
$$

Consider the following constrained optimization problem: Let $H$ be a real Hilbert space. Given a convex objective function $g: H \rightarrow \mathbb{R}$ and $T: H \rightarrow H$ be a nonexpansive mapping such that $\operatorname{Fix}(T) \neq \varnothing$, the problem can be expressed as

$$
\begin{aligned}
& \text { Minimize } g(x) \\
& \text { subject to } x \in F i x(T) \text {. }
\end{aligned}
$$

Optimization problem for a convex objective function over the fixed points set of a nonexpansive mapping have been and will continue to be one of the central problems in nonlinear analysis and is one of the central issues in modern communication networks. Numerous applications in computer vision, machine learning, electronic structure computation, system balancing and robot manipulation can be considered as solving optimization problems. Recently, many iterative algorithms for solving these problems have been proposed, see $[6,2,5,11,8]$ and the references therein.

Very recently, H. Iiduka [7] motivated by the fact that convex optimization problem for a strictly convex objective function over the fixed point set of a nonexpansive mapping includes a network bandwidth allocation problem, which is one of the central issues in modern communication networks, he proposed a fixed point optimization algorithm for solving Problem (1.7).

Algorithm 1.1. Step 0. Choose $x_{0} \in H$ arbitrarily, set $\lambda_{0} \subset(0,1) \quad \alpha_{0}, \subset(0,1]$, and $d_{0}=-\nabla g\left(x_{0}\right)$ arbitrarily and let $n:=0$. Step 1. Given $x_{n} \in H$ and $d_{n} \in H$, choose $\lambda_{n} \subset(0,1), \alpha_{n}, \subset(0,1]$ and compute $x_{n+1} \in K$ as

$$
\left\{\begin{array}{l}
y_{n}=T\left(x_{n}+\lambda_{n} d_{n}\right) \\
x_{n+1}=\alpha_{n} x_{0}+\left(1-\alpha_{n}\right) y_{n}
\end{array}\right.
$$

Step 2. Choose $\beta_{n+1} \in(0,1]$ and compute the direction, $d_{n+1} \in H$, by

$$
d_{n+1}=-\nabla g\left(x_{n}\right)+\beta_{n+1} d_{n} .
$$

Update $n:=n+1$ and go to Step 1 .

Under suitable conditions, he proved that $\left\{x_{n}\right\}_{n \in \mathbb{N}}$ in Algorithm 1.1 weakly converges to a unique solution to Problem (1.7).

Motivated by above results and the fact that the class of demicontractive mappings properly includes that of quasi-nonexpansive, strictly pseudocontractive mappings, we consider the following convex minimization problem : Let $K$ be a nonempty, 
closed and convex subset a real Hilbert space $H$. Given a convex objective function $g: K \rightarrow \mathbb{R}$ be a differentiable, $k$-strongly convex real-valued function. Suppose the differential map $\nabla g: H \rightarrow H$ is $L$-Lipschitz. Let $T_{1}: K \rightarrow K$ be a $\lambda$ demicontractive mapping and $T_{2}: K \rightarrow K$ be a quasi-nonexpansive mapping such that $\Gamma:=\operatorname{Fix}\left(T_{1}\right) \cap \operatorname{Fix}\left(T_{2}\right) \neq \varnothing$. In the present paper, our main purpose is to solve the minimization problem:

$$
\text { find } x^{*} \in \Gamma \text { such that } g\left(x^{*}\right)=\min _{x \in \Gamma} g(x) \text {. }
$$

We denote the set of solutions of Problem (1.9) by $\Omega$.

\section{Preliminaries}

We start with the following demiclosedness principle for nonexpansive mappings.

Lemma 2.1. [1] Let $K$ be a closed convex subset of a real Hilbert space H. Let $T: K \rightarrow K$ be a nonexpansive mapping such that $F(T) \neq \varnothing$. Then $I-T$ is demiclosed at origin.

Lemma 2.2. [3] Let $H$ be a real Hilbert space. Then for any $x, y \in H$, the following inequalities hold:

$$
\begin{gathered}
\|x+y\|^{2} \leq\|x\|^{2}+2\langle y, x+y\rangle . \\
\|\lambda x+(1-\lambda) y\|^{2}=\lambda\|x\|^{2}+(1-\lambda)\|y\|^{2}-(1-\lambda) \lambda\|x-y\|^{2}, \quad \lambda \in(0,1) .
\end{gathered}
$$

Lemma 2.3. [12] Assume that $\left\{a_{n}\right\}$ is a sequence of nonnegative real numbers such that $a_{n+1} \leq\left(1-\alpha_{n}\right) a_{n}+\alpha_{n} \sigma_{n}$ for all $n \geq 0$, where $\left\{\alpha_{n}\right\}$ is a sequence in $(0,1)$ and $\left\{\sigma_{n}\right\}$ is a sequence in $\mathbb{R}$ such that
(a) $\sum_{n=0}^{\infty} \alpha_{n}=\infty$
(b) $\limsup _{n \rightarrow \infty} \sigma_{n} \leq 0$
$r \sum_{n=0}^{\infty}\left|\sigma_{n} \alpha_{n}\right|<\infty$
Then $\lim _{n \rightarrow \infty} a_{n}=0$.

Lemma 2.4. [14] Let $K$ be a nonempty, closed convex subset of a real Hilbert space $H$. Let $A: K \rightarrow H$ be a k-strongly monotone and L-Lipschitzian operator with $k>0, L>0$. Assume that $0<\eta<\frac{2 k}{L^{2}}$ and $\tau=\eta\left(k-\frac{L^{2} \eta}{2}\right)$. Then for each $t \in\left(0, \min \left\{1, \frac{1}{\tau}\right\}\right)$, we have

$$
\|(I-t \eta A) x-(I-t \eta A) y\| \leq(1-t \tau)\|x-y\|, \forall x, y \in K .
$$

Lemma 2.5. [9] Assume $K$ is a closed convex subset of a Hilbert space H. Let $T: K \rightarrow K$ be a self-mapping of $K$. If $T$ is a k-demicontractive mapping, then the fixed point set Fix $(T)$ is closed and convex.

Lemma 2.6. Let $K$ be a nonempty, closed convex subset of a normed linear space E. Let $g: K \rightarrow \mathbb{R}$ a real valued differentiable convex function. Then $x^{*}$ is a minimizer of $g$ over $K$ if and only if $x^{*}$ solves the following variational inequality $\left\langle\nabla g\left(x^{*}\right), y-x^{*}\right\rangle \geq 0$ for all $y \in K$. 
Remark 2.1. By Lemma 2.6, $x^{*} \in \Omega$ if and only if $x^{*}$ solves the following variational inequality problem :

$$
\left\langle\nabla g\left(x^{*}\right), x^{*}-p\right\rangle \leq 0, \forall p \in \Gamma .
$$

We denote the set of solutions of variational inequality problem $(2.1)$ by $V I(\nabla g, \Gamma)$.

\section{Main Results}

In this section, we present our explicit iterative method for solving (1.9).

Lemma 3.1. Let $H$ be a real Hilbert space. Let $K$ be a nonempty, closed convex subset of $H$ and $g: K \rightarrow \mathbb{R}$ be a differentiable, $k$-strongly convex real-valued function. Suppose the differential map $\nabla g: K \rightarrow H$ is L-Lipschitz. Let $T_{1}: K \rightarrow K$ be a $\lambda$-demicontractive mapping and $T_{2}: K \rightarrow K$ be a quasi-nonexpansive mapping such that $\Gamma:=\operatorname{Fix}\left(T_{1}\right) \cap \operatorname{Fix}\left(T_{2}\right) \neq \varnothing$. Then, $\operatorname{VI}(\nabla g, \Gamma)$ is nonempty.

Proof. Set $\eta$ and $\tau$ two real numbers such that $0<\eta<\frac{2 k}{L^{2}}$ and $\tau=\eta\left(k-\frac{L^{2} \eta}{2}\right)$. Let $t_{0}$ be a fixed real number such that $t_{0} \in\left(0, \min \left\{1, \frac{1}{\tau}\right\}\right)$. We observe that $P_{\Gamma}\left(I-t_{0} \eta \nabla g\right)$ is a contraction. Indeed, for all $x, y \in K$, by Lemma 2.4 , we have

$$
\begin{aligned}
\left\|P_{\Gamma}\left(I-t_{0} \eta \nabla g\right) x-P_{\Gamma}\left(I-t_{0} \eta \nabla g\right) y\right\| & \leq\left\|\left(I-t_{0} \eta \nabla g\right) x-\left(I-t_{0} \eta \nabla g\right) y\right\| \\
& \leq\left(1-t_{0} \tau\right)\|x-y\| .
\end{aligned}
$$

Banach's Contraction Mapping Principle guarantees that $P_{\Gamma}\left(I-t_{0} \eta \nabla g\right)$ has a unique fixed point, say $x_{1} \in H$. That is, $x_{1}=P_{\Gamma}\left(I-t_{0} \eta \nabla g\right) x_{1}$. Thus, in view of inequality (1.3), it is equivalent to the following variational inequality problem

$$
\left\langle\nabla g\left(x_{1}\right), x_{1}-p\right\rangle \leq 0, \forall p \in \Gamma .
$$

By using Remark 2.1, we have $x_{1} \in \Omega$. This completes this proof.

We show the main result of this paper, that is, the strong convergence analysis for Algorithm 3.1.

Algorithm 3.1. Step 0. Take $\left\{\alpha_{n}\right\} \subset(0,1),\left\{\theta_{n}\right\}, \subset(0,1),\left\{\beta_{n}\right\} \subset(0,1)$, and $\eta>0$ arbitrarily choose $x_{0} \in K$; and let $n:=0$.

Step 1. Given $x_{n} \in K$, compute $x_{n+1} \in K$ as

$$
\left\{\begin{array}{l}
z_{n}=\theta_{n} x_{n}+\left(1-\theta_{n}\right) T_{1} x_{n}, \\
y_{n}=\beta_{n} z_{n}+\left(1-\beta_{n}\right) T_{2} z_{n}, \\
x_{n+1}=P_{K}\left(I-\eta \alpha_{n} \nabla g\right) y_{n},
\end{array}\right.
$$

Update $n:=n+1$ and go to Step 1 . 
Now we perform the convergence analysis for Algorithm 3.1.

Theorem 3.1. Assume that $I-T_{1}$ and $I-T_{2}$ are demiclosed at origin. Suppose that $\left\{\alpha_{n}\right\},\left\{\theta_{n}\right\}$ and $\left\{\beta_{n}\right\}$ are the sequences such that:

(i) $\lim _{n \rightarrow \infty} \alpha_{n}=0, \quad \sum_{n=0}^{\infty} \alpha_{n}=\infty$

(ii) $\left.\theta_{n} \in\right] \lambda, 1\left[, \lim _{n \rightarrow \infty} \inf \left(1-\theta_{n}\right)\left(\theta_{n}-\lambda\right)>0\right.$ and $\lim _{n \rightarrow \infty} \inf \beta_{n}\left(1-\beta_{n}\right)>0$.

Assume that $0<\eta<\frac{2 k}{L^{2}}$, then, the sequence $\left\{x_{n}\right\}$ defined by Algorithm 3.1 converges strongly to a unique solution of Problem (1.9).

Proof. Firstly, we prove that the sequences $\left\{x_{n}\right\}$ and $\left\{y_{n}\right\}$ are bounded. From Lemma 3.1, we have $V I(\nabla g, \Gamma)$ is nonempty. In what follows, we denote $x^{*}$ to be the unique solution of $V I(\nabla g, \Gamma)$. Without loss of generality, we can assume $\alpha_{n} \in\left(0, \min \left\{1, \frac{1}{\tau}\right\}\right)$ where $\tau=\eta\left(k-\frac{L^{2} \eta}{2}\right)$. Let $p \in \Gamma$. By using (3.1) and Lemma 2.2, we have

$$
\begin{aligned}
\left\|z_{n}-p\right\|^{2}= & \left\|\theta_{n}\left(x_{n}-p\right)+\left(1-\theta_{n}\right)\left(T_{1} x_{n}-p\right)\right\|^{2} \\
= & \theta_{n}\left\|x_{n}-p\right\|^{2}+\left(1-\theta_{n}\right)\left\|T_{1} x_{n}-p\right\|^{2} \\
& -\theta_{n}\left(1-\theta_{n}\right)\left\|T_{1} x_{n}-x_{n}\right\|^{2} .
\end{aligned}
$$

Using the fact that $T_{1}$ is $\lambda$-demi-contractive, we obtain

$$
\begin{aligned}
\left\|z_{n}-p\right\|^{2} \leq & \theta_{n}\left\|x_{n}-p\right\|^{2}+\left(1-\theta_{n}\right)\left(\left\|x_{n}-p\right\|^{2}+\lambda\left\|T x_{n}-x_{n}\right\|^{2}\right) \\
& -\theta_{n}\left(1-\theta_{n}\right)\left\|T_{1} x_{n}-x_{n}\right\|^{2} \\
\leq & \left\|x_{n}-p\right\|^{2}-\left(1-\theta_{n}\right)\left(\theta_{n}-\lambda\right)\left\|T_{1} x_{n}-x_{n}\right\|^{2} .
\end{aligned}
$$

Since $\left.\theta_{n} \in\right] \lambda, 1[$, we have,

$$
\left\|z_{n}-p\right\| \leq\left\|x_{n}-p\right\|
$$

Hence, we obtain

$$
\begin{aligned}
\left\|y_{n}-p\right\| & =\left\|\beta_{n} z_{n}+\left(1-\beta_{n}\right) T_{2} z_{n}-p\right\| \\
& \leq \beta_{n}\left\|z_{n}-p\right\|+\left(1-\beta_{n}\right)\left\|T_{2} z_{n}-p\right\| \\
& \leq\left\|z_{n}-p\right\| .
\end{aligned}
$$

By using inequality (3.2), we have

$$
\left\|y_{n}-p\right\| \leq\left\|z_{n}-p\right\| \leq\left\|x_{n}-p\right\| .
$$

From (3.1), Lemma 2.4 and inequality (3.3), we have

$$
\begin{aligned}
\left\|x_{n+1}-p\right\| & =\left\|P_{K}\left(I-\alpha_{n} \eta \nabla g\right) y_{n}-p\right\| \\
& \leq\left(1-\tau \alpha_{n}\right)\left\|x_{n}-p\right\|+\alpha_{n}\|\eta \nabla g(p)\| \\
& \leq \max \left\{\left\|x_{n}-p\right\|, \frac{\|\eta \nabla g(p)\|}{\tau}\right\} .
\end{aligned}
$$


By induction, it is easy to see that

$$
\left\|x_{n}-p\right\| \leq \max \left\{\left\|x_{0}-p\right\|, \frac{\|\eta \nabla g(p)\|}{\tau}\right\}, \quad n \geq 1
$$

Hence $\left\{x_{n}\right\}$ is bounded also are $\left\{y_{n}\right\}$ and $\left\{\nabla g\left(x_{n}\right)\right\}$.

Consequently, by Lemma 2.4 and inequality (3.2), we obtain

$$
\begin{aligned}
\left\|x_{n+1}-p\right\|^{2} \leq & \left.\|\left(I-\eta \alpha_{n} \nabla g\right)\left(y_{n}-p\right)-\alpha_{n} \eta \nabla g(p)\right) \|^{2} \\
\leq & \alpha_{n}^{2}\|\eta \nabla g(p)\|^{2}+\left(1-\tau \alpha_{n}\right)^{2}\left\|y_{n}-p\right\|^{2} \\
& +2 \alpha_{n}\left(1-\tau \alpha_{n}\right)\|\eta \nabla g(p)\|\left\|y_{n}-p\right\| \\
\leq & \alpha_{n}^{2}\|\eta \nabla g(p)\|^{2}+\left(1-\tau \alpha_{n}\right)^{2}\left\|x_{n}-p\right\|^{2} \\
& -\left(1-\tau \alpha_{n}\right)^{2}\left(1-\theta_{n}\right)\left(\theta_{n}-\lambda\right)\left\|T_{1} x_{n}-x_{n}\right\|^{2} \\
& +2 \alpha_{n}\left(1-\tau \alpha_{n}\right)\|\eta \nabla g(p)\|\left\|x_{n}-p\right\| .
\end{aligned}
$$

Thus,

$$
\begin{array}{r}
\left(1-\tau \alpha_{n}\right)^{2}\left(1-\theta_{n}\right)\left(\theta_{n}-\lambda\right)\left\|T_{1} x_{n}-x_{n}\right\|^{2} \leq\left\|x_{n}-p\right\|^{2}-\left\|x_{n+1}-p\right\|^{2}+\alpha_{n}^{2}\|\eta \nabla g(p)\|^{2} \\
+2 \alpha_{n}\left(1-\tau \alpha_{n}\right)\|\eta \nabla g(p)\|\left\|x_{n}-p\right\| .
\end{array}
$$

Since $\left\{x_{n}\right\}$ is bounded, then there exists a constant $C>0$ such that

$$
\begin{aligned}
\text { (3.4) }\left(1-\tau \alpha_{n}\right)^{2}\left(1-\theta_{n}\right)\left(\theta_{n}-\lambda\right)\left\|T_{1} x_{n}-x_{n}\right\|^{2} \leq & \left\|x_{n}-p\right\|^{2}-\left\|x_{n+1}-p\right\|^{2} \\
& +\alpha_{n} C .
\end{aligned}
$$

Now we prove that $\left\{x_{n}\right\}$ converges strongly to $x^{*}$. We divide the proof into two cases.

Case 1. Assume that the sequence $\left\{\left\|x_{n}-p\right\|\right\}$ is monotonically decreasing sequence. Then $\left\{\left\|x_{n}-p\right\|\right\}$ is convergent. Clearly, we have

$$
\lim _{n \rightarrow \infty}\left[\left\|x_{n}-p\right\|^{2}-\left\|x_{n+1}-p\right\|^{2}\right]=0
$$

It then implies from (3.4) that

$$
\lim _{n \rightarrow \infty}\left(1-\theta_{n}\right)\left(\theta_{n}-\lambda\right)\left\|T_{1} x_{n}-x_{n}\right\|^{2}=0 .
$$

Since $\lim _{n \rightarrow \infty} \inf \left(1-\theta_{n}\right)\left(\theta_{n}-\lambda\right)>0$, we have

$$
\lim _{n \rightarrow \infty}\left\|x_{n}-T_{1} x_{n}\right\|=0
$$

Observing that,

$$
\begin{aligned}
\left\|z_{n}-x_{n}\right\| & =\left\|\theta_{n} x_{n}+\left(1-\theta_{n}\right) T_{1} x_{n}-x_{n}\right\| \\
& =\left\|\theta_{n} x_{n}+\left(1-\theta_{n}\right) T_{1} x_{n}-\theta_{n} x_{n}-\left(1-\theta_{n}\right) x_{n}\right\| \\
& =\left(1-\theta_{n}\right)\left\|T_{1} x_{n}-x_{n}\right\| \\
& \leq\left\|T_{1} x_{n}-x_{n}\right\| .
\end{aligned}
$$


Therefore, from (3.7) we get that

$$
\lim _{n \rightarrow \infty}\left\|z_{n}-x_{n}\right\|=0
$$

Next, we prove that $\limsup _{n \rightarrow+\infty}\left\langle\nabla g\left(x^{*}\right), x^{*}-x_{n}\right\rangle$. Since $H$ is reflexive and $\left\{x_{n}\right\}$ is bounded, there exists a subsequence $\left\{x_{n_{j}}\right\}$ of $\left\{x_{n}\right\}$ such that $x_{n_{j}}$ converges weakly to $a$ in $K$ and

$$
\left.\limsup _{n \rightarrow+\infty}\left\langle\nabla g\left(x^{*}\right), x^{*}-x_{n}\right\rangle=\lim _{j \rightarrow+\infty}\left\langle\nabla g\left(x^{*}\right), x^{*}-x_{n_{j}}\right)\right\rangle .
$$

From (3.7) and $I-T_{1}$ is demiclosed, we obtain $a \in F i x\left(T_{1}\right)$. From Lemma 2.2, the fact that $T_{2}$ is nonexpansive and (3.3), we have

$$
\begin{aligned}
\left\|y_{n}-p\right\|^{2} & =\left\|\beta_{n} z_{n}+\left(1-\beta_{n}\right) T_{2} z_{n}-p\right\|^{2} \\
& =\beta_{n}\left\|z_{n}-p\right\|^{2}+\left(1-\beta_{n}\right)\left\|T_{2} z_{n}-p\right\|^{2}-\left(1-\beta_{n}\right) \beta_{n}\left\|T_{2} z_{n}-z_{n}\right\|^{2} \\
& \leq\left\|x_{n}-p\right\|^{2}-\left(1-\beta_{n}\right) \beta_{n}\left\|T_{2} z_{n}-z_{n}\right\|^{2} .
\end{aligned}
$$

Hence,

$$
\begin{aligned}
\left\|x_{n+1}-p\right\|^{2} \leq & \left\|\left(I-\alpha_{n} \eta \nabla g\right) y_{n}-p\right\|^{2} \\
\leq & \left\|\left(I-\alpha_{n} \eta \nabla g\right)\left(y_{n}-p\right)-\alpha_{n} \eta \nabla g(p)\right\|^{2} \\
\leq & \alpha_{n}^{2}\|\eta \nabla g(p)\|^{2}+\left(1-\alpha_{n} \tau\right)^{2}\left\|y_{n}-p\right\|^{2} \\
& +2 \alpha_{n}\left(1-\alpha_{n} \tau\right)\|\eta \nabla g(p)\|\left\|y_{n}-p\right\| \\
\leq & \alpha_{n}^{2}\|\eta \nabla g(p)\|^{2}+\left(1-\alpha_{n} \tau\right)^{2}\left\|x_{n}-p\right\|^{2} \\
& -\left(1-\alpha_{n} \tau\right)^{2}\left(1-\beta_{n}\right) \beta_{n}\left\|T_{2} z_{n}-z_{n}\right\|^{2} \\
& +2 \alpha_{n}\left(1-\alpha_{n} \tau\right)\|\eta \nabla g(p)\|\left\|x_{n}-p\right\| .
\end{aligned}
$$

Thus, we get

$\left(1-\alpha_{n} \tau\right)^{2} \beta_{n}\left(1-\beta_{n}\right)\left\|T_{2} z_{n}-z_{n}\right\|^{2} \leq\left\|x_{n}-p\right\|^{2}-\left\|x_{n+1}-p\right\|^{2}+\alpha_{n}^{2}\|\eta \nabla g(p)\|^{2}$

$$
+2 \alpha_{n}\left(1-\alpha_{n} \tau\right)\|\eta \nabla g(p)\|\left\|x_{n}-p\right\| \text {. }
$$

Since $\left\{x_{n}\right\}$ is bounded, then there exists a constant $B>0$ sucht that

$$
\left(1-\alpha_{n} \tau\right)^{2} \beta_{n}\left(1-\beta_{n}\right)\left\|T_{2} z_{n}-z_{n}\right\|^{2} \leq\left\|x_{n}-p\right\|^{2}-\left\|x_{n+1}-p\right\|^{2}+\alpha_{n} B .
$$

It then implies from (3.10) and (3.5), that

$$
\lim _{n \rightarrow \infty} \beta_{n}\left(1-\beta_{n}\right)\left\|T_{2} z_{n}-z_{n}\right\|=0 .
$$

Since $\lim _{n \rightarrow \infty} \inf \beta_{n}\left(1-\beta_{n}\right)>0$, we have

$$
\lim _{n \rightarrow \infty}\left\|z_{n}-T_{2} z_{n}\right\|=0
$$


Since $z_{n_{j}} \rightarrow a$, it follows from (3.12) and Lemma 2.1, we have $a \in F i x\left(T_{2}\right)$. Therefore, $a \in \Gamma$. On the other hand, by using $x^{*}$ solves $V I(\nabla g, \Gamma)$, we then have

$$
\begin{aligned}
\limsup _{n \rightarrow+\infty}\left\langle\nabla g\left(x^{*}\right), x^{*}-x_{n}\right\rangle & =\lim _{j \rightarrow+\infty}\left\langle\nabla g\left(x^{*}\right), x^{*}-x_{n_{j}}\right\rangle \\
& =\left\langle\nabla g\left(x^{*}\right), x^{*}-a\right\rangle \leq 0 .
\end{aligned}
$$

Finally, we show that $x_{n} \rightarrow x^{*}$.

$$
\begin{aligned}
\left\|x_{n+1}-x^{*}\right\|^{2} & \leq\left\langle\left(I-\eta \alpha_{n} \nabla g\right) y_{n}-x^{*}, x_{n+1}-x^{*}\right\rangle \\
& \leq\left\langle\left(I-\eta \alpha_{n} \nabla g\right) y_{n}-x^{*}+\eta \alpha_{n} \nabla g\left(x^{*}\right)-\eta \alpha_{n} \nabla g\left(x^{*}\right), x_{n+1}-x^{*}\right\rangle \\
& \leq\left\|\left(I-\alpha_{n} \eta \nabla g\right)\left(y_{n}-x^{*}\right)\right\|\left\|x_{n+1}-x^{*}\right\|+\alpha_{n}\left\langle\eta \nabla g\left(x^{*}\right), x^{*}-x_{n+1}\right\rangle \\
& \leq\left(1-\alpha_{n} \tau\right)\left\|x_{n}-x^{*}\right\|^{2}+2 \alpha_{n} \eta\left\langle\nabla g\left(x^{*}\right), x^{*}-x_{n+1}\right\rangle .
\end{aligned}
$$

From Lemma 2.3, its follows that $x_{n} \rightarrow x^{*}$.

Case 2. Assume that the sequence $\left\{\left\|x_{n}-x^{*}\right\|\right\}$ is not monotonically decreasing sequence. Set $B_{n}=\left\|x_{n}-x^{*}\right\|^{2}$ and $\tau: \mathbb{N} \rightarrow \mathbb{N}$ be a mapping for all $n \geq n_{0}$ (for some $n_{0}$ large enough) by $\tau(n)=\max \left\{k \in \mathbb{N}: k \leq n, B_{k} \leq B_{k+1}\right\}$.

We have $\tau$ is a non-decreasing sequence such that $\tau(n) \rightarrow \infty$ as $n \rightarrow \infty$ and $B_{\tau(n)} \leq B_{\tau(n)+1}$ for $n \geq n_{0}$. From (3.4), we have

$$
\left(1-\theta_{\tau(n)}\right)\left(\theta_{\tau(n)}-\lambda\right)\left\|x_{\tau(n)}-T_{1} x_{\tau(n)}\right\|^{2} \leq \alpha_{\tau(n)} C .
$$

Since $\left.\theta_{\tau(n)} \in\right] \lambda, 1[$, we have

$$
\lim _{n \rightarrow \infty}\left\|x_{\tau(n)}-T_{1} x_{\tau(n)}\right\|^{2}=0 .
$$

By same argument as in case 1 , we can show that $x_{\tau(n)}$ and $y_{\tau(n)}$ are bounded in $H$ and $\left.\lim \sup \left\langle\nabla g x^{*}, x^{*}-x_{\tau(n)}\right)\right\rangle \leq 0$. We have for all $n \geq n_{0}$,

$$
\tau(n) \rightarrow+\infty
$$

$0 \leq\left\|x_{\tau(n)+1}-x^{*}\right\|^{2}-\left\|x_{\tau(n)}-x^{*}\right\|^{2} \leq \alpha_{\tau(n)}\left[-\tau\left\|x_{\tau(n)}-x^{*}\right\|^{2}+2 \eta\left\langle\nabla g x^{*}, x^{*}-x_{\tau(n)+1}\right\rangle\right]$, which implies that

$$
\left\|x_{\tau(n)}-x^{*}\right\|^{2} \leq \frac{2 \eta}{\tau}\left\langle\nabla g x^{*}, x^{*}-x_{\tau(n)+1}\right\rangle .
$$

Then, we have

$$
\lim _{n \rightarrow \infty}\left\|x_{\tau(n)}-x^{*}\right\|^{2}=0
$$

Therefore,

$$
\lim _{n \rightarrow \infty} B_{\tau(n)}=\lim _{n \rightarrow \infty} B_{\tau(n)+1}=0 .
$$

Furthermore, for all $n \geq n_{0}$, we have $B_{\tau(n)} \leq B_{\tau(n)+1}$ if $n \neq \tau(n)$ (that is, $\left.n>\tau(n)\right)$; because $B_{j}>B_{j+1}$ for $\tau(n)+1 \leq j \leq n$. As consequence, we have for all $n \geq n_{0}$,

$$
0 \leq B_{n} \leq \max \left\{B_{\tau(n)}, B_{\tau(n)+1}\right\}=B_{\tau(n)+1} .
$$

Hence, $\lim _{n \rightarrow \infty} B_{n}=0$, that is $\left\{x_{n}\right\}$ converges strongly to $x^{*}$. This completes the proof. 
We now apply Theorem 3.1 for solving convex optimization problems over the set of common fixed point of two nonexpansive mappings without demiclosedness assumption.

Theorem 3.2. Let $H$ be a real Hilbert space and $K$ be a nonempty, closed convex subset of $H$. Let $g: K \rightarrow \mathbb{R}$ be a differentiable, $k$-strongly convex real-valued function and suppose the differential map $\nabla g: K \rightarrow H$ is L-Lipschitz. Let $T_{1}: K \rightarrow K$ and $T_{2}: K \rightarrow K$ two nonexpansive mappings such that $\Gamma:=$ Fix $\left(T_{1}\right) \cap$ Fix $\left(T_{2}\right) \neq \varnothing$. Assume that $0<\eta<\frac{2 k}{L^{2}}$. Let $\left\{x_{n}\right\}$ be a sequence defined iteratively from arbitrary $x_{0} \in K$ by:

$$
\left\{\begin{array}{l}
z_{n}=\theta_{n} x_{n}+\left(1-\theta_{n}\right) T_{1} x_{n}, \\
y_{n}=\beta_{n} z_{n}+\left(1-\beta_{n}\right) T_{2} z_{n}, \\
x_{n+1}=P_{K}\left(I-\eta \alpha_{n} \nabla g\right) y_{n},
\end{array}\right.
$$

Suppose that $\left\{\alpha_{n}\right\},\left\{\theta_{n}\right\}$ and $\left\{\beta_{n}\right\}$ are the sequences such that:

(i) $\lim _{n \rightarrow \infty} \alpha_{n}=0, \quad \sum_{n=0}^{\infty} \alpha_{n}=\infty$

(ii) $\left.\theta_{n} \in\right] 0,1\left[\right.$, and $\lim _{n \rightarrow \infty} \inf \beta_{n}\left(1-\beta_{n}\right)>0$. Then, the sequence $\left\{x_{n}\right\}$ defined by (3.14) converges strongly to a minimizer of $g$ over Fix $\left(T_{1}\right) \cap$ Fix $\left(T_{2}\right)$.

Proof. Since every nonexpansive mapping is quasi-nonexpansive and 0-demicontractive. The proof follows Lemma 2.1 and Theorem 3.1.

We apply Theorem 3.1 for solving the following quadratic optimization problem:

$$
\text { find } x^{*} \in \Gamma \text { such that } g\left(x^{*}\right)=\min _{x \in \Gamma} g(x), \text { where } g(x)=\frac{1}{2}\langle A x, x\rangle \text {. }
$$

Theorem 3.3. Let $H$ be a real Hilbert space and $K$ be a nonempty, closed convex subset of $H$. Let $A: K \rightarrow H$ be strongly bounded linear operator with coefficient $k>0$. Let $T_{1}: K \rightarrow K$ be a $\lambda$-demicontractive mapping and $T_{2}: K \rightarrow K$ be a quasi-nonexpansive mapping such that $\Gamma:=F i x\left(T_{1}\right) \cap F i x\left(T_{2}\right) \neq \varnothing$. Assume that $0<\eta<\frac{2 k}{\|A\|^{2}}$. Let $\left\{x_{n}\right\}$ be a sequence defined iteratively from arbitrary $x_{0} \in K$ by:

$$
\left\{\begin{array}{l}
z_{n}=\theta_{n} x_{n}+\left(1-\theta_{n}\right) T_{1} x_{n}, \\
y_{n}=\beta_{n} z_{n}+\left(1-\beta_{n}\right) T_{2} z_{n} \\
x_{n+1}=P_{K}\left(I-\eta \alpha_{n} A\right) y_{n}
\end{array}\right.
$$

Assume that $I-T_{1}$ and $I-T_{2}$ are demiclosed at origin. Suppose that $\left\{\alpha_{n}\right\},\left\{\theta_{n}\right\}$ and $\left\{\beta_{n}\right\}$ are the sequences such that: 
(i) $\lim _{n \rightarrow \infty} \alpha_{n}=0, \quad \sum_{n=0}^{\infty} \alpha_{n}=\infty$,

(ii) $\left.\theta_{n} \in\right] \lambda, 1\left[, \quad\right.$ and $\lim _{n \rightarrow \infty} \inf \beta_{n}\left(1-\beta_{n}\right)>0$. Then, the sequence $\left\{x_{n}\right\}$ defined by (3.16) converges strongly to a solution of problem (3.15).

Proof. The proof follows Theorem 3.1 and Remark 1.1 with $\nabla g x=A x$.

Now, we give some remarks on our results as follows:

(1) Our results improve many recent results using fixed point optimization algorithm to approximate minimizers of convex functions over the set of common fixed points of nonlinear mappings.

(2) Our results are applicable for solving variational inequality problems involving strongly monotone and Lipschitzian operator and fixed point problems involving quasi-nonexpansive and demicontractive mappings.

\section{REF E R E N C E S}

1. F. E. BRowder: Convergenge theorem for sequence of nonlinear operator in Banach spaces, Math.Z.100(1967) 201-225. Vol. EVIII, part 2, 1976.

2. P.L. Combettes: A block-iterative surrogate constraint splitting method for quadratic signal recovery, IEEE Transactions on Signal Processing 51(7), 1771-1782 (2003).

3. C. E. Chidume: Geometric Properties of Banach spaces and Nonlinear Iterations, Springer Verlag Series: Lecture Notes in Mathematics, 1965,(2009), ISBN 978-184882-189.

4. B. Halpern: Fixed points of nonexpansive maps, Bull. Amer. Math. Soc., 3 (1967), 957-961.

5. I. Hideaki: Convergence Analysis of Iterative Methods for Nonsmooth Convex Optimization over Fixed Point Sets, Mathematical Programming, October (2015).

6. I. HIDEAKI: Fixed point optimization algorithm and its application to power control in CDMA data networks, Math. Program. 133 (2012) 227-242.

7. I. HIDEAKI: Fixed point optimization algorithm and its application to network bandwidth allocation, Journal of Computational and Applied Mathematics, 236 (2012), 1733-1742.

8. I. Hideaki, I. Yamada: A use of conjugate gradient direction for the convex optimization problem over the fixed point set of a nonexpansive mapping, SIAMJ.Optim. 19 (2009), 1881-1893.

9. G. MARino, H.K. XU: Weak and strong convergence theorems for strict pseudocontractions in Hilbert spaces, J. Math. Math. Appl., 329 (2007), 336-346.

10. A. MoudAFi: Viscosity approximation methods for fixed-point problems, J. Math. Anal. Appl. 241 (2000), 46-55. 
11. M. Ulbrich: Semismooth Newton Methods for Variational Inequalities and Constrained Optimization Problems in Function Space, in: MPS-SIAM Series on Optimization, 2011.

12. H.K. XU: Iterative algorithms for nonlinear operators, J. London Math. Soc. 66 (2002), no. 2, $240-256$.

13. Y. YaO, H. Zhou, Y. C. Liou: Strong convergence of modified Krasnoselskii-Mann iterative algorithm for nonexpansive mappings, J. Math. Anal. Appl. Comput. 29 (2009) 383-389.

14. S. WANG: A general iterative method for an infinite family of strictly pseudocontractive mappings in Hilbert spaces, Applied Mathematics Letters, 24 (2011): 901 - 907. 